\title{
Determination of pedestrian's personal space in mass religious gatherings - A case study of Kumbh Mela
}

\author{
Ms. Aparna P M, Ms. Karthika P Sobhana, Dr. Ashish Verma \\ Department of Civil Engineering \\ Indian Institute of Science \\ Bangalore, India \\ aparna.pras@gmail.com; pskarthika@iisc.ac.in; ashishv@iisc.ac.in
}

\begin{abstract}
Personal space can be defined as the physical distance between two individuals in a social environment. It varies from person to person depending on culture and context and there are significant individual differences too. Studying personal space includes the ability to recognize the various zones of involvement and the activities, relationships, and emotions associated with each zone. This paper tries to formulate and define personal space in high density crowd situations in Kumbh Mela, one of the world's largest mass religious gatherings. Video data of pilgrims taking part in the Panchkroshi Yatra, a religious walkathon which is a part of KumbhMela, is used for the analysis of factors affecting personal space. To identify the thresholds of personal space, walking speed of individuals, gender, presence of luggage and the number of males and females surrounding an individual have been considered. It is found that the average speed of the individual, the group size, and the gender ratio of group members have a significant effect on the personal space of an individual. Also, it is observed that the personal space follows an asymmetrical pattern rather than a symmetrical pattern.
\end{abstract}

Keywords: Inter-personal distance, Personal space, Mass religious gatherings, Stampede, Psychology

\section{Introduction}

Personal space is defined as the immediate space available to an individual for exercising his movements without causing discomfort to oneself and his/her immediate neighbour. Most of the literature on personal space either treats the personal space as circular in nature on the lines of [2] or studies the personal space intrusions at a psychological level from a medical perspective. [9] has defined personal space as the flight zone of a human with respect to other humans and the person steps away to reinstate the margin of safety when their personal space is violated. The complexity of personal space comes from the fact that its dimensions are affected by many factors like the context, personal relation, status, gender, culture, intentions etc. The concept of personal space is rooted in zoology and ecology [5], but it is an entirely different concept from that of territory.

In this study, our aim is to understand and define the concept of personal space in a mass religious gathering, especially at high density conditions. Kumbh Mela, where an expected 100 million people visits the area in a span of one month, is taken as the study scenario. The event exhibits different crowd scenarios ranging from low density single file movement to a tightly compressed torrent of pedestrians. The crowd scenario studied here consists of pedestrian flow in a confined space, where masses of people are trying to get to the destination with a defined purpose.

While moving in a dense crowd, pedestrians adjust both speed and step length in such a way as to avoid collisions and to preserve their personal space. Personal space invasion is likely to increase physiological stresses and therefore individuals tend to maintain personal space zones to protect themselves. As far as a mass religious gathering is concerned, it is important not to have personal space intrusions since it can leave a threat for potential stampede, which may be caused due to possible 
aggression. This study, thus, ascertains how behavioural and psychological aspects affect crowd situations, and the relevance of studying interpersonal distances in the context of mass religious gatherings.

The upcoming section discusses the motivation behind pursuing the study. Data used for the study as well as analysis of personal space intrusions is discussed in Section 3. The last section concludes with the important findings of the study.

\section{Background and Motivation}

Humans tend to maintain a certain distance from others as they are walking. This interpersonal distance is important as it creates a protective buffer zone in a human mind. It is natural to feel discomfort when other people intrude upon this safe bubble. It is more pronounced when the intruders are strangers. This feeling of uneasiness causes psychological stress and in the case of a mass gathering where it is unavoidable, this uneasiness can cause defensive reflexes. It is imperative that these local disturbances do not affect the crowd and result in unfortunate incidents.

Over the last four decades, anthropologists and psychologists theorized that personal space was a zone maintained by individuals to keep others at a comfortable distance during different social interactions ([5],[12], [2]).Studies have also concentrated on personal space as a protective zone during locomotion that provides sufficient time to perceive environmental hazards, plan gait adaptations, and execute them. There are no studies about the way people use space in high density crowds.

The effect of intercultural differences on personal space has received a fair attention from fields like social psychology, where the aim is to understand the differences in personal space between different ethnic groups. [4] interviewed people from different cultural backgrounds and shows that Anglo Saxons, and Asians uses larger personal space than Caucasians or Latinos.[2] described personal space as the region surrounding an individual where a person can touch or hold or grasp others with their arms extended, this is generally a distance of $45-75 \mathrm{~cm}$. [10] found that proxemic distance is greater in the rear $(\sim 120 \mathrm{~cm})$ than in the front and on the sides $(\sim 60 \mathrm{~cm}$ each) using an experiment conducted with students. While [1] found that personal space is bigger in the front than in the rear, which even drops to zero in the rear if test subjects are not allowed to move their heads ([8],[11]).

[6] hypothesizes that as the number of pedestrians increases, interpersonal distances reduce, and the walking group follows an inverted U-shape. Also, as the number of pedestrians increases, the number of direction changes while walking increases. However, most of the work reported is in situations of low density. The spatial pattern of the groups with respect to the alignment within the group, group size, group composition, and the effect of these proxemic variables on walking speed is investigated by [3]. It is observed that higher walking speeds are correlated with the mis-alignment within the groups. $>$ formation, stair disposition, < formation prevailed more in groups compared to the aligned spatial arrangement. It is also noted that males preserve more interpersonal distance when compared to females. However, majority of these studies refrain from using locations of excessive social density, and hence fail to study the effect of changes in density on personal space violations and walking behavior.

[7] explores the possibility of using a virtual environment rather than a physical experimental setup to study the personal space of adults while they were trying to circumvent a cylindrical obstacle in their path. However, most of the existing literatures study personal space within a laboratory setup, with controlled experimental conditions. The situation is vastly different in field where hundreds of people walk for long distances in extreme weather conditions, and the slightest provocation can result in unforeseen incidents. Therefore, the main focus of this paper is to understand the shape of personal space around an individual in a field setting, and to ascertain if it is symmetrical in nature. Further, an attempt is 
made to understand the variables that are likely to influence the personal space intrusions in Kumbh Mela.

\section{Data description and analysis}

\subsection{Data description}

Video data of pilgrims moving in the Ambodhya Stretch, which is a part of Panchkroshi Yatra, spanning five days was analysed. It is an annual religious walkathon where pilgrims take a circuitous route of $118 \mathrm{~km}$ around the city covering many temples and finally take a holy bath in the sacred river, in a limited span of five days. Fig 1 shows the screenshots of the study section.

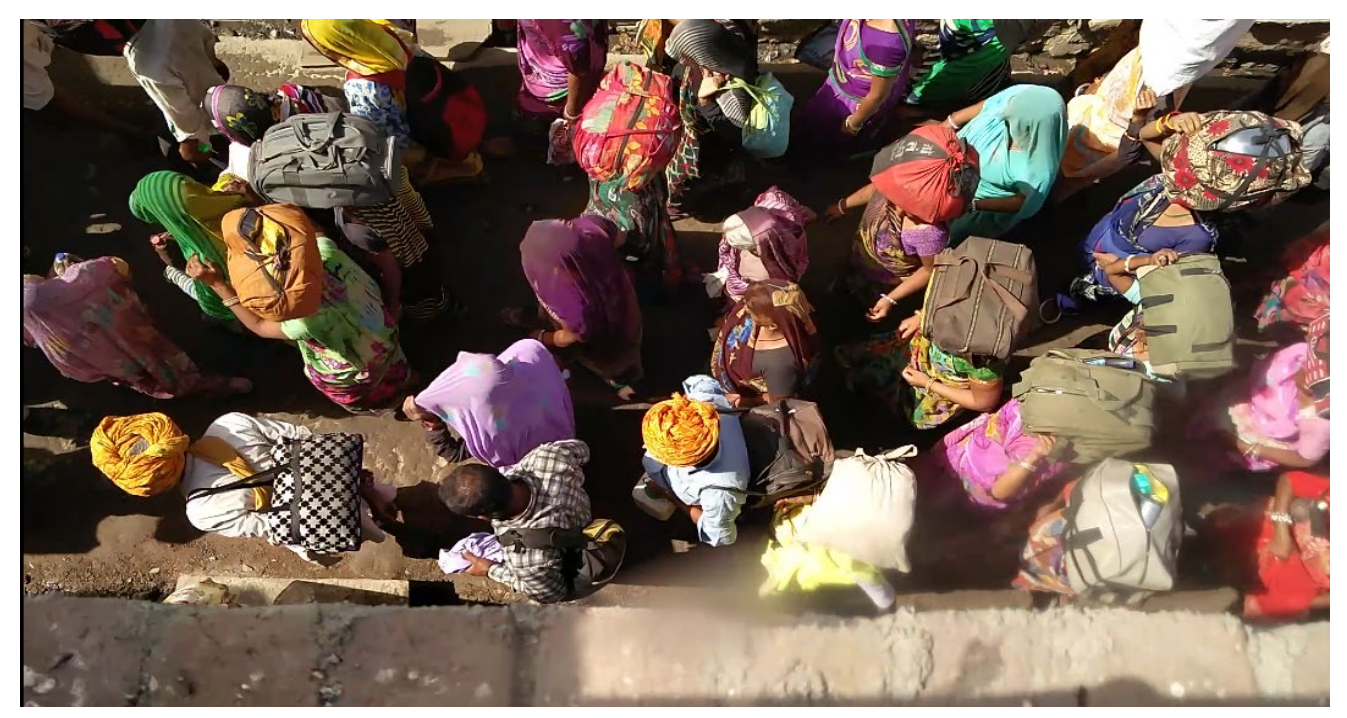

Fig. 1: Snapshot of the study section (Ambodhya stretch)

A 15 minutes long video of unidirectional flow was observed, and a total of 102 groups were identified for analysis. The Traffic Data Extractor developed by IIT Bombay was used to track pedestrians. A central person was identified and then all the persons who were in the immediate vicinity of the central person were also identified. A group is then defined as this collection of central person and the surrounding persons.

The central person within each group, gender of the central person and his/her neighbours, and the presence of luggage was additionally noted down. Of these 726 persons, 538 were females $(74.10 \%)$ and 188 were males $(25.89 \%)$ and out of those people, 569 persons were carrying luggage (78. 37\%). The entire sample was segregated into two groups; female centric group, and male centric group. Of this, almost $85 \%$ of the female centric group, and $55 \%$ of male centric group had head baggage.

In many literatures, personal space is also perceived as a protective zone during walking: it allows people to perceive dangers and adapt their walking strategies to alleviate those hazards. Here, personal space is defined as the immediate space surrounding a person and enclosed by boundaries created by his/her immediate neighbours. This is based on the hypothesis that in a large mass religious gathering as Kumbh Mela, people are aware of the magnitude of the gathering. In such high densities, the concern to personal space intrusion would be the immediate surrounding rather than the people at the periphery. It is also hypothesized that the shape of the personal space would not be circular, mostly because the pedestrian flow is unidirectional. 


\subsection{Analysis and Results}

A group of pedestrians are identified from video, with the aim to analyze the personal space requirements of the pedestrian positioned at the centre of each group. The analysis of the personal space bubble as defined by the space around the central person is done from the time the central person enters the frame till the time the person exits the frame. The personal space around a person mostly follows an oblong asymmetrical pattern rather than a symmetrical one. This could be the result of the unidirectional flow of pedestrians along the walkway as hypothesized earlier. It is necessary to preserve a larger distance in the front rather than rear to ensure a comfortable walking gait. It is also observed that the central person tries to maintain the initial distance with all neighbours as they continue to walk through the section.

Frequency distribution of personal space area

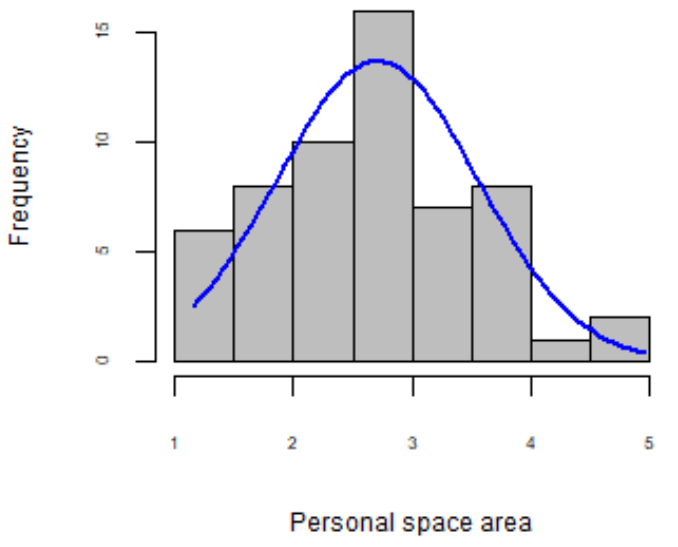

Fig. 2: Histogram of personal space area

Average personal space area of an individual is calculated as the area of the polygon obtained by connecting the coordinates of immediate neighbours. Fig 3 shows the frequency distribution of personal space area. The personal space follows a normal distribution with a mean value of $2.71 \mathrm{~m}^{2}$. Personal space area ranges from $1.15 \mathrm{~m}^{2}$ to $4.96 \mathrm{~m}^{2}$. The effect of the individual's average speed, the number of neighbours, gender of the central person, ratio of same gender to the opposite gender in the group members, and presence of luggage on the personal space is modelled using linear regression. The parameter estimates, and t-stats are presented in Table 1.

Table 1: Regression model parameter estimates

\begin{tabular}{|l|c|c|}
\hline \multicolumn{1}{|c|}{ Explanatory variable } & Parameter estimate & $\begin{array}{c}\text { Significanc } \\
\text { e level }\end{array}$ \\
\hline Constant & $-2.61(-3.63)$ & $* * *$ \\
\hline Average speed of individual & $2.79(4.62)$ & $* * *$ \\
\hline Number of immediate neighbours & $0.51(7.08)$ & $* * *$ \\
\hline Opposite gender to same gender indicator & $0.62(3.12)$ & $* *$ \\
\hline
\end{tabular}




\begin{tabular}{|c|c|c|}
\hline Presence of head luggage & $0.20(1.04)$ & - \\
\hline $\mathrm{R}^{2}$ & \multicolumn{2}{|c|}{0.60} \\
\hline Adjusted $\mathrm{R}^{2}$ & \multicolumn{2}{|c|}{0.52} \\
\hline Number of observations & \multicolumn{2}{|c|}{60} \\
\hline
\end{tabular}

Based on the t-statistics obtained, it could be concluded that all the parameter estimates, except for head luggage, are found to be statistically significant at $95 \%$ confidence level, as all the t-stats are found to be greater than the $t_{\text {crit }}$ value of 1.96. As per the model, the average speed parameter estimate gives the marginal impact of the variable on the personal space, given everything else remains the same. For every unit increase in average speed, the personal space is expected to increase by 2.79 units, ceteris paribus. The gender of the central person was found to be negative, which indicates that females are willing to stay closer when compared to males. The same result has been reported from previous studies conducted in experimental settings. Head luggage was found to be insignificant. However, the variable is retained in the model as the authors feel that the variable contributes to a great extent in deciding the personal space. Another variable considered was an indicator variable which indicates the ratio of number of group members with opposite gender (with respect to the central person) to the number of group members with same gender. This variable was found to be significant and it indicates that people try to maintain a larger personal space when opposite gender dominates the surrounding group.

However, the personal space requirements are likely to differ if the surrounding people are familiar. In such large gatherings, people may come together and stay as a group. Also, familiarity implies that they move around together. However, identifying such groups from video data is a difficult task. Common indicators used in identifying people belonging to a group include hand holding, eye contact, sharing a luggage between each other, or holding on to the same child. These interchanges between people are used as indicators but extracting such information even under medium density conditions is not trivial. In the recent past, clustering techniques have been employed to detect abnormal crowd flow patterns [14], group trajectories [13], and detection and tracking of dense crowds. The popularity of cluster analysis is that technique can cluster objects such that the members of each cluster are closer to each other than members of other cluster.

Here, social groups are identified through clustering technique. The trajectory information is used to identify social groups in each frame using K-means clustering technique, a standard clustering approach. In this clustering technique, the data points are grouped into $k$-clusters (where $k$ is the defined number of clusters). Here, the clustering is done by minimizing the Euclidean distance between the data points and the corresponding centroids. However, the clustering is sensitive to the initial position of cluster centroids. The optimal number of clusters is determined using the elbow method, where the point of inflection in the plot of sum of squared errors (SSE) for each value of $k$ is taken to be optimal- $k$. Further, social groups identified as per the visual observations are used to validate the identified clusters. Voronoi diagrams are constructed to delineate the clusters visually, such that each cell in the region corresponds to one social group. Such proximity diagrams are used quite frequently, wherein each of the cells is convex polygons, and this is further used to identify the personal space within each cell/group. Figure 3 shows the identified clusters for a few sample frames. 


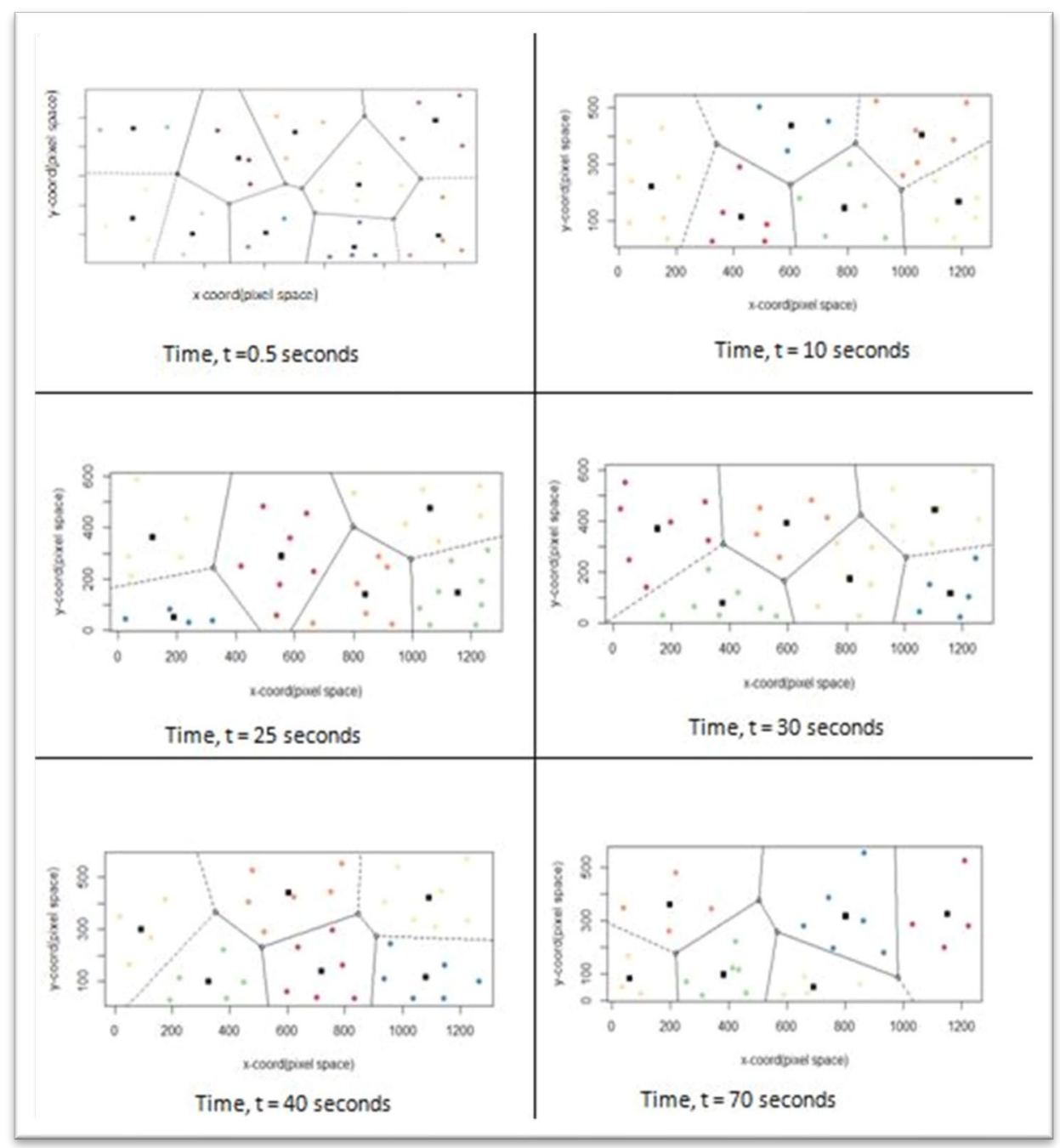

Fig. 3: Voronoi plots of clusters for different time frames

Groups obtained are categorized as female-dyads, female-triads, female-quartets, and male-triads. It is seen that there is significant difference in the mean personal space requirements of all-female groups compared to all-male groups. (female-dyads: $0.26 \mathrm{~m}$, female-triads: $0.23 \mathrm{~m}$, female-quartets: $0.23 \mathrm{~m}$, and male-triads: $0.16 \mathrm{~m}$ ). This could be the result of several factors including the nature of the event, the crowd being female-dominated, and due to the differences between experimental and field conditions.

\section{Conclusion}

The aim of this study was to define and understand the concept of personal space in a mass religious gathering. The analysis of trajectories of groups of people around a central person revealed that the shape of the bubble surrounding a person, i.e. the personal space is not symmetrical in nature. The bubble tapers in the longitudinal direction while maintaining approximately the same distance laterally on either side. This refutes the assumptions of circular personal space as reported in earlier literatures, especially in situations of medium density. It is also noted that as walking speed increases, personal space increases. This could in turn be the result of lower densities, which permit people to walk at a higher pace. A new insight from this study is the effect of the dominant gender group on the personal space. 
There is a lot of heterogeneity in the way people perceive personal space across different cultures and different backgrounds. In Kumbh Mela, a mix of urban, rural, and foreign population, either singly or in groups of different sizes, participate in multiple religious activities together. This heterogeneity reflects in how individuals set rules of personal space for themselves. For this reason, it is extremely challenging to model the personal space as perceived by an individual. It is in this context that the results from this study can be used as inputs to simulate pedestrian behaviour in large crowds.

Further work would include studying the variation of personal space in different locations and varying densities. Group composition is found to influence personal space. Taking this forward, the work can be extended to study the interrelation between group behavior and personal space in higher densities.

\section{Acknowledgements}

The work reported in this paper is part of the project titled "The Kumbh Mela Experiment: Measuring and Understanding the Dynamics of Mankind's largest crowd," funded by the Ministry of Electronics and IT Ministry of Communication and Information Technology, Government of India (MITO-0105), Netherlands Organization for Scientific Research, NWO (Project no. 629.002.202), and Robert Bosch Center for Cyber Physical Systems, Indian Institute of Science, Bangalore. (Grant No. RBCO001). The authors also express their gratitude towards Kumbh Mela administration and government of Madhya Pradesh, India for providing constant support and official permissions to carry out research work and establish Indo-Dutch collaboration research camp at Kumbh Mela 2016.

The authors also acknowledge the help rendered by Mr Abhinav Gyan, intern at IISc Bangalore, during data extraction for this study.

\section{References}

[1] L. A. Hayduk, "The shape of personal space: An experimental investigation.," Canadian Journal of Behavioural Science/Revue canadienne des sciences du comportement, vol. 13, no. 1, pp. 87-93, 1981.

[2] E. T. Hall, "The Hidden Dimension," Leonardo, vol. 6, no. 1, p. 94, 1973.

[3] M. Costa, "Interpersonal Distances in Group Walking," Journal of Nonverbal Behavior, vol. 34, no. 1, pp. 15-26, Apr. 2009.

[4] C. Beaulieu, "Intercultural Study of Personal Space: A Case Study," Journal of Applied Social Psychology, vol. 34, no. 4, pp. 794-805, 2004.

[5] R. Sommer, "Studies in Personal Space," Sociometry, vol. 22, no. 3, p. 247, 1959.

[6] A. Frohnwieser, R. Hopf, and E. Oberzaucher., "Human Walking Behavior - The Effect of Pedestrian Flow and Personal Space Invasions on Walking Speed and Direction", Human Ethology Bulletin, vol. 28, no. 3, pp. 20-28, 2013.

[7] M. Gérin-Lajoie, C. L. Richards, and B. J. Mcfadyen, "The Negotiation of Stationary and Moving Obstructions during Walking: Anticipatory Locomotor Adaptations and Preservation of Personal Space," Motor Control, vol. 9, no. 3, pp. 242-269, 2005.

[8] L. A. Hayduk, "The permeability of personal space.," Canadian Journal of Behavioural Science/Revue canadienne des sciences du comportement, vol. 13, no. 3, pp. 274-287, 1981.

[9] M. S. Graziano and D. F. Cooke, "Parieto-frontal interactions, personal space, and defensive behavior," Neuropsychologia, vol. 44, no. 6, pp. 845-859, 2006.

[10] R. C. Newman and D. Pollack, "Proxemics in deviant adolescents.," Journal of Consulting and Clinical Psychology, vol. 40, no. 1, pp. 6-8, 1973.

[11] L. A. Hayduk and S. Mainprize, "Personal Space of the Blind," Social Psychology Quarterly, vol. 43, no. 2, p. 216, 1980. 
[12] K. B. Little, "Personal space," Journal of Experimental Social Psychology, vol. 1, no. 3, pp. 237-247, 1965.

[13] R. Sharma, and T. Guha, "A trajectory clustering approach to crowd flow segmentation in videos", 2016 IEEE International Conference on Image Processing (ICIP),2016.

[14] Ma, Jian, and Weiguo Song. "Automatic Clustering Method of Abnormal Crowd Flow Pattern Detection." Procedia Engineering 62 ,509-18. 2013 